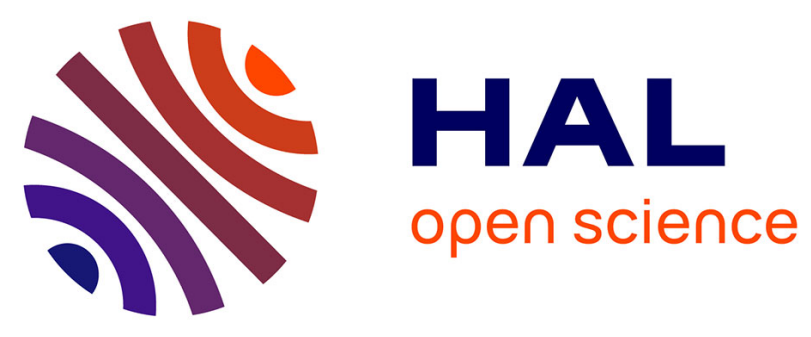

\title{
Reclassifying inconclusive diagnosis after newborn screening for cystic fibrosis. Moving forward
}

Aurelie Hatton, Anne Bergougnoux, Katarzyna Zybert, Benoit Chevalier, Myriam Mesbahi, Jean Pierre Altéri, Katarzyna Walicka-Serzysko, Magdalena Postek, Magali Taulan, Aleksander Edelman, et al.

\section{To cite this version:}

Aurelie Hatton, Anne Bergougnoux, Katarzyna Zybert, Benoit Chevalier, Myriam Mesbahi, et al.. Reclassifying inconclusive diagnosis after newborn screening for cystic fibrosis. Moving forward. Journal of Cystic Fibrosis, 2021, 10.1016/j.jcf.2021.12.010 hal-03503341

\section{HAL Id: hal-03503341 https://hal.science/hal-03503341}

Submitted on 28 Dec 2021

HAL is a multi-disciplinary open access archive for the deposit and dissemination of scientific research documents, whether they are published or not. The documents may come from teaching and research institutions in France or abroad, or from public or private research centers.
L'archive ouverte pluridisciplinaire HAL, est destinée au dépôt et à la diffusion de documents scientifiques de niveau recherche, publiés ou non, émanant des établissements d'enseignement et de recherche français ou étrangers, des laboratoires publics ou privés. 


\title{
Reclassifying inconclusive diagnosis after newborn screening for cystic fibrosis. Moving forward
}

\author{
Aurelie Hatton ${ }^{\mathrm{a}, \mathrm{b}}$, Anne Bergougnoux ${ }^{\mathrm{c}, \mathrm{d}}$, Katarzyna Zybert ${ }^{\mathrm{e}}$, Benoit Chevalier ${ }^{\mathrm{a}, \mathrm{b}}$, \\ Myriam Mesbahi ${ }^{\mathrm{a}, \mathrm{b}}$, Jean Pierre Altéri ${ }^{\mathrm{d}}$, Katarzyna Walicka-Serzysko ${ }^{\mathrm{e}}$, Magdalena Postek ${ }^{\mathrm{e}}$, \\ Magali Taulan-Cadars ${ }^{\mathrm{c}, \mathrm{f}}$, Aleksander Edelman ${ }^{\mathrm{a}, \mathrm{b}}$, Alexandre Hinzpeter ${ }^{\mathrm{a}, \mathrm{b}}$, Mireille Claustres ${ }^{\mathrm{f}}$, \\ Emmanuelle Girodon ${ }^{\mathrm{a}, \mathrm{g}}$, Caroline Raynal ${ }^{\mathrm{c}, \mathrm{d}}$, Isabelle Sermet-Gaudelus ${ }^{\mathrm{a}, \mathrm{b}, \mathrm{h}, \mathrm{i}, *,}$, Dorota Sands ${ }^{\mathrm{e}}$ \\ a INSERM U1151, Institut Necker Enfants Malades, Université de Paris, 149 rue de Sévres, Paris 75015, France \\ b Université de Paris, Paris, France \\ ' PhyMedExp, INSERM U1046, CNRS UMR 9214, University of Montpellier, Montpellier, France \\ ¿ CHU de Montpellier, Laboratoire de Génétique Moléculaire, Montpellier, France \\ e Cystic Fibrosis Department, Institute of Mother and Child, Warsaw, Poland \\ ${ }^{\mathrm{f}}$ Université de Montpellier, Montpellier, France \\ g Laboratoire de Génétique et Biologie Moléculaires, Hôpital Cochin, APHP Centre, Université de Paris, Paris, France \\ ${ }^{\mathrm{h}}$ Centre de Référence Maladies Rares, Mucoviscidose et maladies apparentées, Hôpital Necker Enfants Malades, Paris, France \\ i European Reference Network-Lung, France
}

Keywords:

Cystic fibrosis

CFTR

Neonatal screening

Functional tests

\begin{abstract}
A B S T R A C T
Background: Newborn screening for Cystic Fibrosis (CF) is associated with situations where the diagnosis of CF or CFTR related disorders (CFTR-RD) cannot be clearly ruled out.

Materials/patients and methods: We report a case series of 23 children with unconclusive diagnosis after newborn screening for CF and a mean follow-up of 7.7 years (4-13). Comprehensive investigations including whole CFTR gene sequencing, in vivo intestinal current measurement (ICM), nasal potential difference (NPD), and in vitro functional studies of variants of unknown significance, helped to reclassify the patients.

Results: Extensive genetic testing identified, in trans with a CF causing mutation, variants with varying clinical consequences and 3 variants of unknown significance (VUS). Eighteen deep intronic variants were identified by deep resequencing of the whole CFTR gene in 13 patients and were finally considered as non-pathogenic. All patients had normal CFTR dependent chloride transport in ICM. NPD differentiated 3 different profiles: CF-like tracings qualifying the patients as CF, such as F508del/D1152H patients; normal responses, suggesting an extremely low likelihood of developing a CFTR-RD such as F508del/TG11T5 patients; partial CFTR dysfunction above $20 \%$ of the normal, highlighting a remaining risk of developing CFTR-RD such as F508del/F1052V patients. The 3 VUS were reclassified as variant with defective maturation (D537N), defective expression (T582I) or with no clinical consequence (M952T).

Conclusion: This study demonstrates the usefulness of combining genetic and functional investigations to assess the possibility of evolving to CF or CFTR-RD in babies with inconclusive diagnosis at neonatal screening.
\end{abstract}

\section{Introduction}

Newborn screening (NBS) for Cystic Fibrosis (CF) is associated with situations where the diagnosis of CF cannot be clearly ruled out $[1,2]$. The prevalence of these unconcluded situations, also

* Corresponding author at: INSERM U1151, Institut Necker Enfants Malades, Université de Paris, 149 rue de Sévres, Paris 75015, France.

E-mail address: Isabelle.sermet@aphp.fr (I. Sermet-Gaudelus). called Cystic Fibrosis Screen Positive Inconclusive Diagnosis (CFSPID) is variable according to the population and the screening algorithm, ranging from 1 to $6 \%$ in Australia and Canada to $11 \%$ in France [3] and 21\% in California [4].

A number of studies have followed up CFSPID babies and evaluated the advent of $\mathrm{CF}$ [3-13] This paper focus on the evolution of CFSPID to CFTR-related disorder (CFTR-RD) later in life, in babies carrying compatible genotypes. This diagnostic dilemma and the uncertainty of evolution make the management of these chil- 
dren challenging. This also causes parents to face a great deal of anxiety about their infants' prognosis and uncertain future [14].

Usually, those patients have an intermediate sweat test between 30 and $60 \mathrm{mmol} / \mathrm{L}$. In a number of cases, genetic testing identifies variants of unknown clinical significance (VUS) and variants with varying clinical consequence (VVCC), which, in trans with a CF-causing mutation, may cause either CF or a CFTR-RD $[15,16]$. To increase interpretation complexity, a number of these variants show an incomplete penetrance for the different phenotypes, which makes their clinical relevance challenging [17]. This is the case of $\mathrm{R} 117 \mathrm{H} \mathrm{[18]}$ or the $5 \mathrm{~T}$ allele of the poly-T tract in intron 8 whose severity is related to the associated TG repeat [19]. Thus, there is an urgent need to implement a strategy aiming to detect infants who might convert to CF or a CFTR-RD in order to propose appropriate follow up and possible access to CFTR modulators.

We report a case series of 23 children with unconclusive diagnosis after NBS for CF. All carried a CF-causing variant in trans of a VVCC or a VUS. Comprehensive investigations including whole CFTR gene sequencing, in vivo functional experiments by intestinal current measurement (ICM) and nasal potential difference (NPD), along with in vitro functional studies of VUS, led to reclassify the patients. This classification was related to clinical follow-up.

\section{Methods}

\subsection{Population}

Patients were referred for confirmation of diagnosis for CF after detection, at neonatal screening of an IRT above the 99.4 percentile and two CFTR variants identified within the 3-tier-screening Polish program (IRT/DNA/DNA sequencing) $[20,21]$ The first sequencing step includes sequencing of exons:7, 10, 11, 13, 17B, 18, 20, 21, fragment of intron 19 to identify $3849+10 \mathrm{kbC}>\mathrm{T}$ and search for dele 2,3(21 kb). The second step in case a variant is identified includes sequencing of exon 1, 2, 3, 4, 5, 6, 8, 9, 12, 14A, 14B, 15, 16, $17 \mathrm{~A}, 19,22,23$ and 24 , search for $5 \mathrm{~T}$ and TG repeat.

3524660 newborns were screened between 2006 and 2016 in the newborn screening catchment area of Warsaw. 165 were diagnosed as CF; 89 were diagnosed as CRMS/CFSPID (including IVS8TG11T5, TG11T5 thereafter) or 52 (without TG11T5). These 23 cases were from the 89 who accepted the investigation. Parents gave the consent to participate in that study .

Patients were enrolled if they presented an intermediate sweat test and carried 2 variants with at least one VVCC or if their genotype included a VUS. They all underwent regular clinical and microbiological follow-up every 3 months according to existing standard of care including NPD and ICM [22]. The mean clinical followup was 7.7 years (4-13). The patients were informed about functional tests and data collection was made following the regulations described by the Declaration of Helsinki and the Huriet-Sérusclat law on biomedical research ethics. This study was approved by the Bioethics Committee at the Institute of Mother and Child, Warsaw.

\subsection{Whole CFTR gene sequencing, bioinformatics analysis}

A whole CFTR gene analysis was carried out in all patients in order to find other potential pathogenic variants undetected by DNA sequencing included in the NBS program. Libraries of single stranded DNA fragments were prepared using a Nextera Rapid Capture custom design (Illumina, San Diego, CA, USA), covering the whole CFTR locus on chromosome 7q32, and sequencing of the libraries was carried out on a MiSeq instrument (Illumina) according to the manufacturer's protocol (detailed protocol in Supplemental materials). Secondary analysis and annotation of the variants was performed using our in-house pipeline Nenufaar [23].
We classified CFTR variants regarding clinical, genetics and functional data reported in CFTR1 [24], CFTR2 and CFTR-France [25] databases. CFTR2 categorizes variants as CF causing, VVCC or non-CF-causing. CFTR-France helps differentiate among VVCC (i) those with an increased penetrance for CF, (ii) those with a low penetrance for $\mathrm{CF}$ and a high penetrance for CFTR-RD, and (iii) those with a low penetrance for CFTR-RD, when they are frequently observed in healthy individuals in trans with a CF-causing variant. Missense VUS were analyzed by CYSMA [26] to predict their pathogenic impact on the protein and by data aggregator MobiDetails [27]. CFTR deep-intronic variants of potential interest were prioritized by Nenufaar and analyzed using three different splicing predictors (details in Supplemental materials) and were entered in MobiDetails to assess their Variant Allele Frequency (V.A.F.) frequency in the general population (gnomAD) and find potential reports in general databases (e.g., dbSNP, ClinVar).

\subsection{In vitro studies to assess synthesis and maturation of the CFTR protein}

We used expression vector pTracer-CMV containing the CFTRwild-type (WT) cDNA, provided by Pr. Fanen, to assess the impact of VUS [28]. pTracer-CFTR constructs (WT and mutants) were transfected into HeLa cells ( $n=4$ independent transfections) to quantify CFTR protein expression and maturation by western blot experiments (mature form, $180 \mathrm{kD}-\mathrm{C}$ band and immature non glycosylated form, $150 \mathrm{kD}-\mathrm{B}$ band) (detailed protocol in Supplemental materials).

\subsection{In vitro studies to assess the function of the CFTR protein}

CFTR activity was measured in transiently transfected HEK293 cells (with pTracer-CFTR WT and mutant constructs) using halidesensitive yellow fluorescent protein YFP-H148Q/I152L [29]. This reflects CFTR activity as the Iodide ( $\mathrm{I}^{-}$) anion transported by CFTR quenches the fluorescent probe (e.g.; decrease in fluorescence in response to cAMP is indicative of CFTR activity) (detailed protocols in Supplementary materials).

\subsection{Intestinal current measurement (ICM) and nasal potential difference (NPD)}

ICM was performed to investigate CFTR $\mathrm{Cl}^{-}$transport. NPD was additionally performed in patients who did not accept ICM and to better characterize patients carrying VUS or VVCC, particularly those with unknown penetrance for CFTR-RD. ICM and NPD were performed according to the European Cystic Fibrosis Society Clinical Trials Network (ECFS-CTN) standard operating procedures [30,31]. All NPD and ICM tests were interpreted by two investigators, blinded to the clinical characteristics of the patients.

Briefly, CFTR activity was assessed in the rectal epithelium by the IsC change upon Forskolin ( $\Delta$ Forskolin) which evaluates the cAMP dependent active transport, and by the ICM Derichs score $\Delta$ Forskolin $+\Delta$ Carbachol $+\Delta$ Histamine. CFTR function in the nasal epithelium was determined based on the NPD variation in response to Amiloride perfusion in high Chloride $\left(\mathrm{Cl}^{-}\right)$solution ( $\triangle$ Amiloride); and the sum of the changes in NPD after perfusion with the low $\mathrm{Cl}^{-}$solution and isoproterenol ( $\Delta$ Total $\mathrm{Cl}^{-}$Response $\triangle T C R)$. Normal values were derived from previous values in healthy controls $\left(\Delta\right.$ Forskolin $=16.6(14) \mu \mathrm{A} / \mathrm{cm}^{2} ; \Delta \mathrm{TCR}=-18(11)$ $\mathrm{mV}$ and $\Delta$ Amiloride $=7.2(5) \mathrm{mV})[30,31]$. 
Table 1

Summary of clinical, genetic and functional data at the moment of the test of 23 patients presenting with unconclusive diagnosis at neonatal screening for $\mathrm{CF}$.

\begin{tabular}{|c|c|c|c|}
\hline & \multicolumn{3}{|l|}{ Genotypes } \\
\hline & $\begin{array}{l}\text { CF / VVCC or VVCC / VVCC (CF } \\
\text { or CFTR-RD) }\end{array}$ & $\begin{array}{l}\text { CF / VVCC CFTR-RD-low } \\
\text { penetrance }\end{array}$ & CF / VUS \\
\hline Number of patients & 12 & 7 & 4 \\
\hline Age, Mean (min-max), years & $5.1(1-10)$ & $4(1.5-10)$ & $4.8(3-8)$ \\
\hline IRT ng/ml, Mean (min-max) & $215(105-370)$ & $169(145-239)$ & $151(89-225)$ \\
\hline $\begin{array}{l}\text { Sweat chloride concentration }(\mathrm{mmol} / \mathrm{L}) \\
\text { Mean (min-max) }\end{array}$ & $38(30-58)$ & $34(26-45)$ & $22(16-36)$ \\
\hline Number of patients with sputum production & 6 & 1 & 0 \\
\hline Number of patients with $\mathrm{PI}^{*}$ & 0 & 0 & 0 \\
\hline Lung imaging & $\begin{array}{l}\text { Wall thickening }(n=4) \\
\text { mucus plugging }(n=2)\end{array}$ & Wall thickening $n=2$ & Not reported \\
\hline Bronchial colonization & S.aureus $(n=4)$ & $n=0$ & $n=1$ \\
\hline $\begin{array}{l}\text { Number of patients with respiratory } \\
\text { symptoms suggestive of CF } 3 \text { years later }\end{array}$ & $n=2$ & $n=0$ & $n=0$ \\
\hline$\Delta$ Forskolin, $(\mu \mathrm{A} / \mathrm{cm} 2)$ & $61(39-87)$ & $55(30-95)$ & $51(46-59)$ \\
\hline Mean (min-max) & $n=9$ & $n=6$ & $n=4$ \\
\hline \multicolumn{4}{|l|}{ Number of patients } \\
\hline Derichs score, Median (min-max) & $261(181-434)$ & $257(134-345)$ & $240(208-269)$ \\
\hline Detailed genotypes (number of patients if & F508del/D1152H $(n=3)$ & R553X/TG11T5 $(n=2)$ & F508del/D537N \\
\hline \multirow[t]{7}{*}{$n>1)$} & F508del/R117H;T7 $(n=2)$ & F508del/ TG11T5 $(n=4)$ & $3849+10 \mathrm{kbC}>\mathrm{T} / \mathrm{T} 582 \mathrm{I}$ \\
\hline & R553X/TG12T5 & F508del/R31C & F508del/M952T $(n=2)$ \\
\hline & F508del/F1052V & & \\
\hline & F508del/R1162L $(n=2)$ & & \\
\hline & N1303K/R1162L & & \\
\hline & F508del/L997F & & \\
\hline & TG12T5/TG13T5 & & \\
\hline
\end{tabular}

CFTR-RD: CFTR-related disorders; *PI: Pancreatic Insufficiency, based on pancreatic elastase $<200 \mu \mathrm{g} / \mathrm{g}$ of stool; VVCC: Varying Clinical Significance; VUS: variants of unknown significance.

\section{Results}

\subsection{Patients}

Clinical, genetic and functional data of the cohort of infants are summarized in Table 1 . Mean follow up of the patients was 7.7 years (4-13). IRT varied from 89 to $370 \mathrm{ng} / \mathrm{ml}$. Nineteen patients had a sweat test between 30 and $60 \mathrm{mmol} / \mathrm{L}$ (mean sweat test: $37 \mathrm{mmol} / \mathrm{L}$ (range: $30-58 \mathrm{mmol} / \mathrm{L}$ ), while 4 , carrying a VUS (D537N, T582I, and M952T) or a TG11T5 variant in trans of a CF-causing variant had a normal sweat test below $30 \mathrm{mmol} / \mathrm{L}$. At the time of investigation, mean age of the patients was 4.7 years (range: 1-10 years). All patients were pancreatic sufficient, based on elastase $>200 \mu \mathrm{g} / \mathrm{g}$ of stools. S.aureus was found in sputum of 5 patients. Three years later, the three patients carrying F508del/D1152H (P7, P8, P9) demonstrated sputum production, wall thickening and bronchial wall thickening with bronchiectasis for P8. Sweat test was assessed in 15 out the 23 patients at the 3 year follow up. Two patients (P1, F508del/R117H;7T and P5, TG12T5/TG13T5) displayed conversion of the sweat chloride above $60 \mathrm{mmol} / \mathrm{L}$ and were categorized as CF. Three additional patients increased significantly their sweat chloride (P2, R553X/TG12T5; P7 and P9 (both F508del/D1152H)) while 4 patients decreased the chloride sweat value below $30 \mathrm{mmol} / \mathrm{l}$ (P4 and P10, both F508del/R1162L; P16 and P23, both F508del/TG11T5). The remaining patients displayed no significant changes (Supplementary Table S1)

\subsection{Genetic testing}

One patient was TG12T5/TG13T5 (VVCC/VVCC) and 22 carried a CF-causing variant (F508del $n=17$, R553X $n=3$; N1303K $n=1$; $3849+10 \mathrm{kbC}>\mathrm{T} n=1$ ) combined with a non CF-causing variant on the other parental allele. Final classification of this 2nd variant was based on CFTR2 and CFTR-France as explained in Supplementary Table S2. Three patients carried the D1152H VCC variant which displays an increased penetrance for CF; 4 carried a VCC variant with increased penetrance for CFTR-RD (TG12T5, TG13T5, $\mathrm{R} 117 \mathrm{H} ; \mathrm{T} 7, \mathrm{~F} 1052 \mathrm{~V}$ ); 3 patients carried variants categorized as non CF-causing (L997F, R1162L) in CFTR2 but considered as CFTR-RD causing in the CFTR-France database; 7 patients carried TG11T5 $(n=6)$ and $\mathrm{R} 31 \mathrm{C}(n=1)$. Those variants are associated with low penetrance for CFTR-RD, according to their prevalence in the general population [13]; 4 patients carried a VUS (D537N $(n=1)$, T582I $(n=1)$ or M952T $(n=2)$.

Whole CFTR gene sequencing identified on average 200 variants per patient. After secondary analysis and prioritization by Nenufaar, we focused on 18 deep intronic variants identified in 13 different patients (Supplementary Table S3) either because they had a V.A.F. lower than 0.01 suggestive of a potential pathogenic effect $(n=17)$ and/or in silico predictions suggestive of a possible impact on splicing $(n=5)$. The c. $1209+425 \mathrm{C}>$ T variant was very unlikely to be pathogenic because of its high frequency in the general population $(>0.01)$. Several variants were probably in complex allele (i.e., on the same chromosome) with exonic variants, as they displayed the same frequency in the general population. The variants c.54-10943G $>A$ and c.1393-1489_1393-1488del were probably in cis with $\mathrm{R} 117 \mathrm{H}$, and c.2620-3342A $>G$ in cis with R553X $(n=2)$. None of these deep-intronic variants was associated with additional pathogenic consequence, based on the clinical or the functional data collected in the infants.

\subsection{CFTR in vivo or ex vivo functional evaluation of patients carrying VVCC}

Nineteen patients underwent ICM and demonstrated a CFTR dependent $\mathrm{Cl}^{-}$transport in the normal range based on $\Delta$ Forskolin and the ICM score (Table 1, Supplementary Table S1, Supplementary Fig. S1). Those 4 patients who did not have ICM underwent NPD, which was reliable in 3 of them (P8, P9, P16). Two carried the F508del/D1152H genotype (P8, P9). NPD showed a decreased cAMP dependent $\mathrm{Cl}^{-}$transport in the $\mathrm{CF}$ range and a high response 
to Amiloride (Supplementary Fig. S2, Panel A). The third one carried the TG11T5 allele in trans with R553X and showed normal values (P16) (Supplementary Fig. S2, Panel B). Additionally, NPD was also performed in the patient carrying the F508del/F1052V genotype (P3). This patient had a normal CFTR dependent $\mathrm{Cl}^{-}$transport but an elevated response to Amiloride, indicative of CFTR dysfunction (Supplementary Fig. S2, Panel C).

Altogether, the combination of the genetic and the functional information helped categorize these patients. Four patients were categorized as Pancreatic sufficient CF because of consistent conversion of sweat chloride above $60 \mathrm{mmol} / \mathrm{L}(n=2 ; \mathrm{P} 1$ F508del/R117H;7T and P5 TG12T5/TG13T5), or NPD in the CF range (P8 and P9, both carrying the F508del/D1152H genotype). The third F508del/D1152H patient (P7) was classed at high likelihood to develop CF because of high penetrance of $\mathrm{D} 1152 \mathrm{H}$ for $\mathrm{CF}$. The 14 remaining patients had a normal ICM for 12 of them (not performed for the last 2). The patients carrying the R31C or the TG11T5 variant (P16 to P23) were classed at low risk to develop CFTR-RD because of the low penetrance of these variants for CFTR-RD while the others were considered at risk for developing CFTR-RD later in life because of high penetrance of their VVCC for CFTR-RD (P2, P3, P4, P6, P10, P11, P12) (Supplementary Table S1).

\subsection{Characterization of patients carrying variants of unknown significance}

Four patients of the cohort carried a VUS (1609G $>A$, p.Asp537Asn, D537N; c.1745C > T, p. Thr582Ile, T582I; c.2855T>C, p.Met952Thr, M952T) in trans of F508del $(n=3)$ or $3849+10 \mathrm{kbC}>\mathrm{T}(n=1)$ (Table 1 , Supplementary Table S1). The main 3D structures of the mutants and their consequences are described in Supplementary Fig. S3 and Supplementary Table S4.

The D537N variant displays $100 \%$ conservation among CFTR orthologs but is present in only $6.88 \%$ sequences of NBD1 homologs (Supplementary Table S4, Supplementary Fig. S3). D537N showed a significant impact on the quantity of total CFTR protein $(B+C$ ratio (mean \pm SD) of $33 \% \pm 23$ of the WT) and a moderate impact on CFTR maturation as shown by the decreased quantity of mature mutant protein, based on the $C /(B+C)$ ratio of $83 \% \pm 6 \%$ (Fig. $1 C$ and D). It strongly decreases CFTR activity to $14 \% \pm 6$ of the WT. This residual function is partially potentiated by VX-770 up to $30 \%$ \pm 5 (Fig. 1E, Supplementary Fig. 4). The patient carrying D537N in trans with F508del had normal CFTR dependent $\mathrm{Cl}^{-}$transport in the intestinal mucosa in contrast to the nasal mucosa where NPD showed a high response to Amiloride while $\mathrm{Cl}^{-}$transport was normal (Fig. 1A and B). This enabled us to categorize this variant as a CFTR-RD causing variant, similar to F1052V.

The T582I variant, also located in NBD1, displays $100 \%$ conservation among CFTR orthologs and $22.68 \%$ conservation among NBD1 homologs (Supplementary Table S4, Supplementary Fig. S3). Western blot experiments showed a mild impact on the quantity of the CFTR protein with a B $+C$ ratio of $58 \% \pm 19$ but no significant impact on CFTR maturation (Fig. $2 \mathrm{~B}$ and $\mathrm{C}$ ). The halide-sensitive functional test showed a decreased CFTR activity at $39 \% \pm 13$ of the WT which was restored to $64 \% \pm 8$ by VX-770 (Fig. 2D). The CFTR dependent $\mathrm{Cl}^{-}$transport was normal in the intestinal mucosa of the patient carrying T582I in trans with $3849+10 \mathrm{kbC}>\mathrm{T}$ (Fig. 2A, Supplementary Fig. S4).

The M952T variant is $100 \%$ conserved among orthologs and $51.97 \%$ among MSD2 homologs. In vitro experiments show that this mutant does not significantly impact protein formation or function (Fig. 3-D) and thus should be considered to be of no clinical consequence. The two patients, carrying M952T in trans of F508del had normal $\mathrm{Cl}^{-}$transport in the intestinal mucosa (Fig. 3A, Supplementary Fig. S4).

\section{Discussion}

This case series of inconclusive cases after NBS for CF investigates the usefulness of a combination of genetic and functional investigations to assess the likelihood of progressing to CF or CFTR$\mathrm{RD}$ in asymptomatic newborns.

We provide here mainly functional insights and some relation with clinical evolution for some genotypes such as $\mathrm{D} 1152 \mathrm{H}$. The polish neonatal screening programs was initiated in region of Warsaw in 2006 and on a nationwide basis in 2009. Therefore, the main limitation of this study is a follow-up time relatively short (mean follow up of 7.7 years, ranging between 4 years and 13 years). Thus the advent of CFTR-RD in these subjects cannot be fully assessed.

Extensive genetic screening led to identify, in trans with a CF causing mutation, variants that were initially classified as VVCC. Further consideration of their penetrance for CF or CFTR-RD based on systematic analysis of CFTR2 and CFTR-France as well as their frequency in the general population allowed us to reclassify them as (i) VVCC with an increased penetrance for CF (D1152H); (ii) VVCC with an increased penetrance for CFTR-RD and a low penetrance for CF (R117H;7T, TG12T5; TG13T5; F1052V, L997F, R1162L), and (iii) VVCC with a low penetrance for CFTR-RD (TG11T5 and R31C), concordant with previous studies $[17,18]$. L997F, R1162L are classed as non CF-causing in CFTR2 but are considered as CFTRRD causing in CFTR-France, based on epidemiological data, which led us to reclassify them as VVCC. The case of the TG12T5/TG13T5 patient is very illustrative. This patient displayed a conversion of sweat test about $60 \mathrm{mmol} / \mathrm{l}$ at the 3 years follow up illustrating the impact of the 5T variant in association with a TG12 or TG13 repeat in babies with unconclusive diagnosis at NBS when in trans with a CF causing mutation. Importantly, the $5 \mathrm{~T}$ variant is not searched in other traditional NBS programs using IRT/DNA panel algorithms and therefore, those babies are "missed".

The genetic classification was extended by whole CFTR gene studies to search for additional CFTR pathogenic deep-intronic variants that would not have been found by conventional sequencing techniques [32,33]. All the new variants found were finally considered as non-pathogenic per se, considering infants' phenotypes, concordance with their previously determined genotype, functional investigations, in silico predictions and the notable frequency in the general population.

Interestingly, all the patients in this study had a Forskolin response above $50 \%$ of the normal ratio. This is probably because CFTR shows a high level of expression in the intestinal mucosa. Therefore, as the transepithelial transport reflects both channel dysfunction and the number of proteins expressed, a high number of CFTR channels displaying a residual activity might result in a globally normal intestinal transepithelial transport. Indeed, in the murine G480C CF animal model, levels of WT-protein as low as $20 \%$ were sufficient to normalize transepithelial anion secretion [34]. Thus, it can be hypothesized that a normal ICM Forskolin response indicates a CFTR function at least above $20 \%$ and suggests that those patients are at very low risk of developing typical CF disease, but does not rule out a CFTR-RD. In this context, evaluation of CFTR function in the respiratory mucosa brings additional information as it evaluates CFTR activity based on $\mathrm{Cl}^{-}$transport and its impact on ENaC activity [31].

Our measurements individualized 3 situations. First, tracings showing a typical CF profile as observed in the F508del/D1152H patients, indicating a high likelihood of developing CF. This was supported by the fact that the patients had developed CF like symptoms 3 years later. Unfortunately, we could not perform ICM in these patients. However, it is known that patients carrying $\mathrm{D} 1152 \mathrm{H}$ in trans of a CF causing variant develop CF although less severe than F508del, putting this VVCC in the CF range [35]. More- 
A

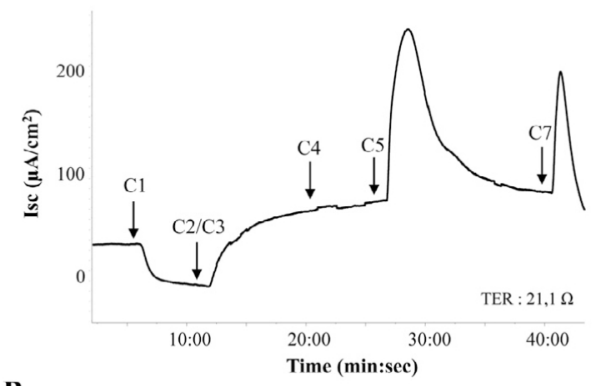

B

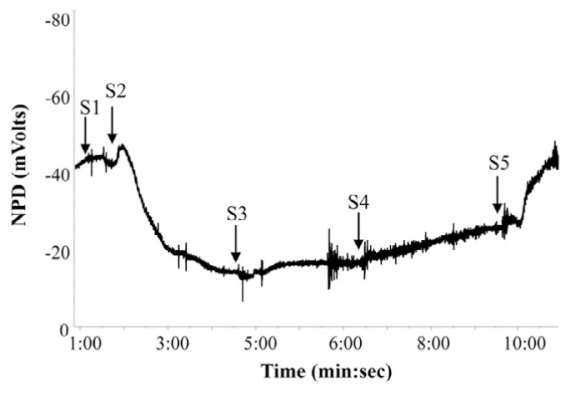

C

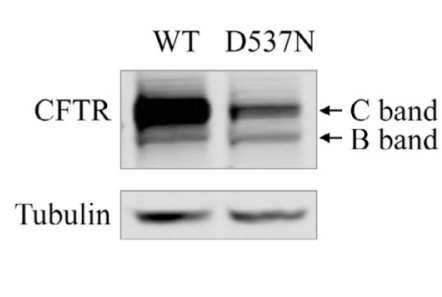

D

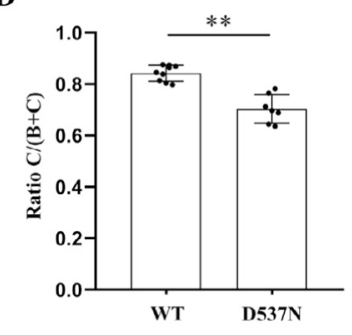

$\mathbf{E}$

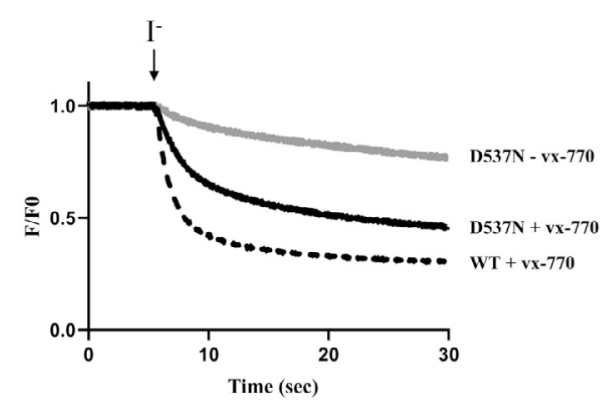

Fig. 1. c.1609G >A - p.Asp537Asn (D537N) CFTR expression and functional experiments A. Short-circuit current (Isc) change of rectal biopsy of Basal IsC (C1) after successive

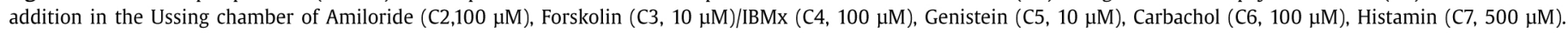
B. Nasal potential difference (NPD) tracing. Baseline PD is measured after perfusion of the nasal epithelium with saline Ringer solution (S1). PD changes are recorded after:perfusion with $100 \mu \mathrm{M}$ amiloride in Ringer solution (S2), $100 \mu \mathrm{M}$ amiloride in low-chloride solution (S3), $100 \mu \mathrm{M}$ amiloride plus $10 \mu \mathrm{M}$ isoproterenol in low-chloride solution (S4), $100 \mu \mathrm{M}$ amiloride plus $10 \mu \mathrm{M}$ isoproterenol plus $100 \mu \mathrm{M}$ ATP in low-chloride solution (S5). C. Western blot analysis of WT and D537N CFTR. Fully glycosylated ( $C$ band) and core glycosylated CFTR (B band) are indicated with an arrow. Tubulin was probed to assess equal loading amounts. D. Histograms showing the ratio of $C$ band on total CFTR. Data are shown as mean + - S.D. $n=9$ for WT and $n=7$ for D537N with ${ }^{* *}$ indicating $p<0.01$ (Kruskal-Wallis test). E. Iodide efflux experiments in HEK cells expressing D537N-CFTR after perfusion with CPT-CAMP $(100 \mu \mathrm{M})$ without (in gray) or with VX770 $(10 \mu \mathrm{M}$, in black). Iodide efflux experiments in HEK cells transfected with WT-CFTR after perfusion with CPT-cAMP $(100 \mu \mathrm{M})$ and VX-770 $(10 \mu \mathrm{M})$ indicated as a reference (black dashed line).

A

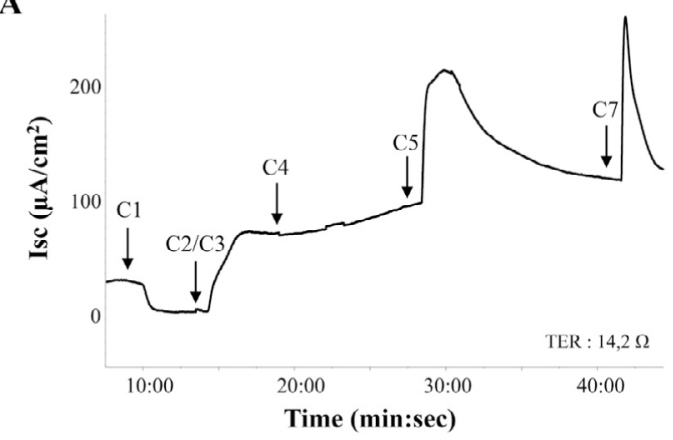

C

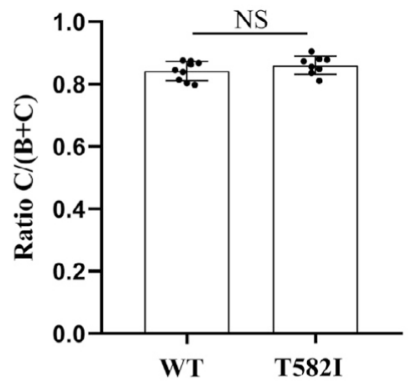

B
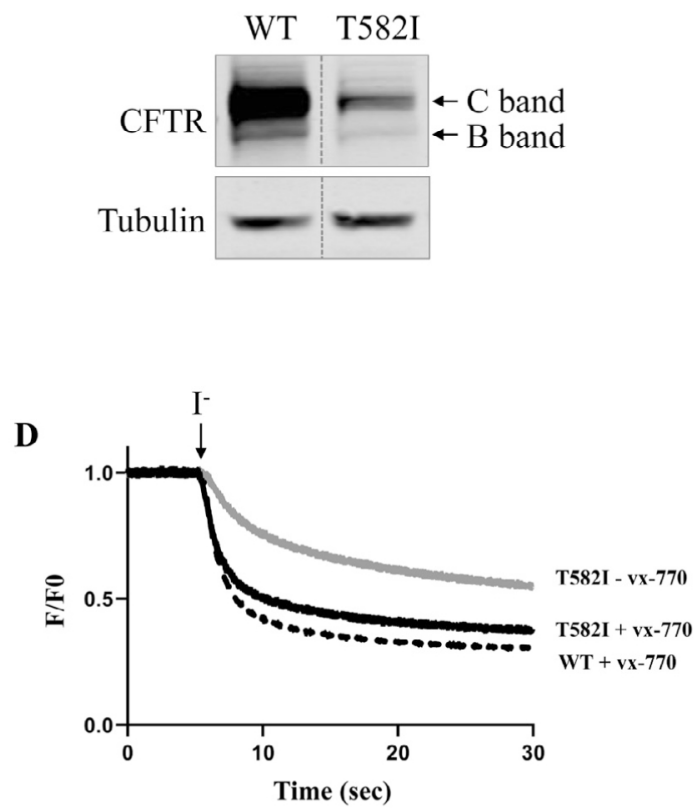

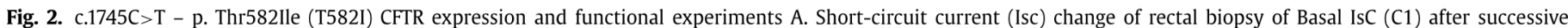

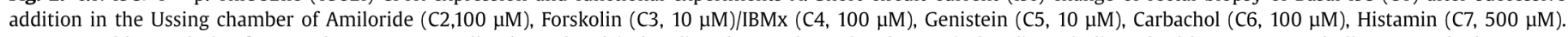

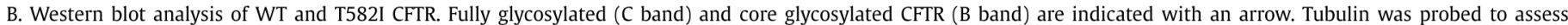

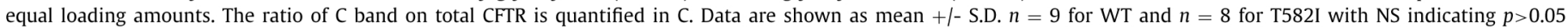

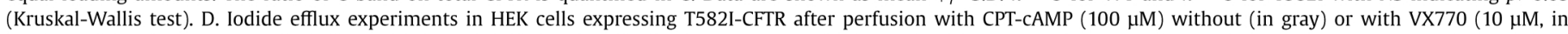

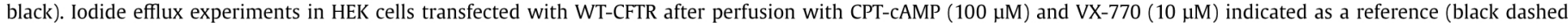
line). 
A
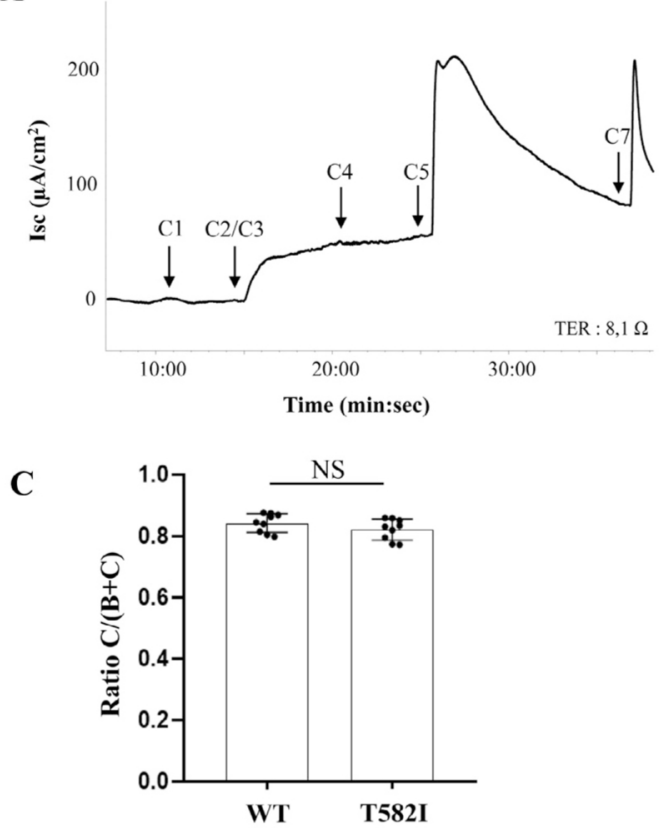

B
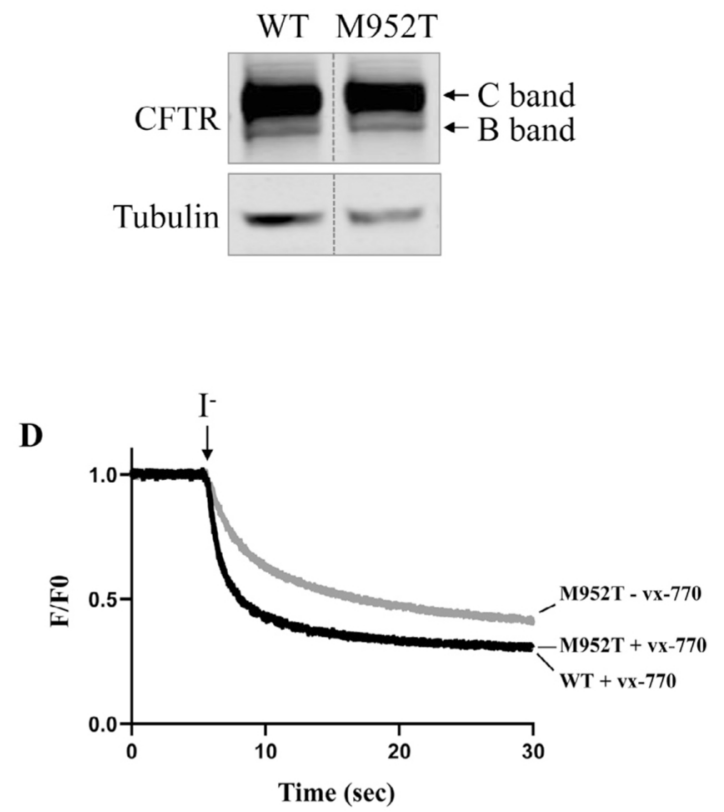

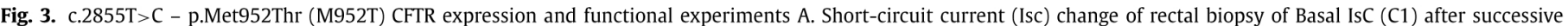

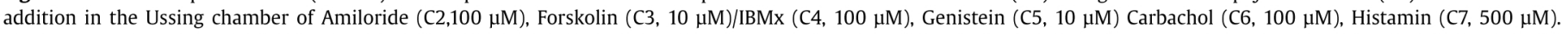

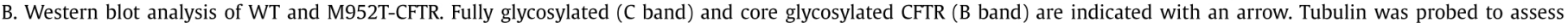

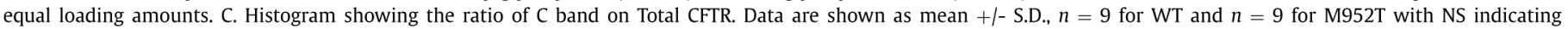

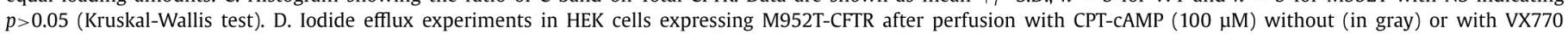

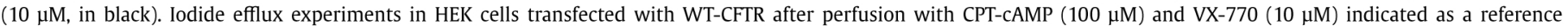
(black dashed line).

over, Minso et al. reported a CFTR activity in the CF range in intestine in a D1152H homozygous patient [36], Second, tracings with responses in the normal range in patients carrying variants with low penetrance for CFTR-RD, such as TG11T5. This suggests that these patients are at an extremely low risk, if any, of developing a CFTR-RD. Consistently, no CF symptoms were reported in these patients at a mean age of 8 years. This confirms the observation by Aalbers et al. that the TG11T5 variant is associated with lower sweat chloride, less frequently abnormal NPD and less progression of symptoms compared to TG12T5 and TG13T5 variants $[13,37]$. This observation might be translated to the patients carrying $\mathrm{R} 31 \mathrm{C}$ and more generally for variants with a very low penetrance for CFTR-RD based on their frequency in the general population $[17,38]$. Third, and finally, patients with NPD tracings showing an increased response to Amiloride but a normal or subnormal $\mathrm{Cl}^{-}$transport, such as in the patient carrying F508del in trans with F1052V or D537N. Similar profiles with an isolated ENaC hyperactivity have already been reported in patients carrying CFTR-RD mutations and were significantly improved by Ivacaftor treatment $[11,39]$. These results, together with the observation of ICM in the normal range, suggest a partial CFTR dysfunction above $20 \%$ of the WT but a remaining possibility of developing CFTR-RD in these patients.

Three very rare variants were fully characterized for the first time. In vitro functional studies by western blot experiments showed normal maturation and halide transport assay for M952T which suggests a normal function. However, this mutant has been associated with CBAVD in combination with a CF-causing variant. This suggests that this variant could have some pathogenic effect, but with a low penetrance for CFTR-RD [40]. The 2 patients carrying this variant in trans of F508del were thus considered at very low risk to develop CFTR-RD. In contrast, T582I displayed a moderate defect in CFTR expression but a defective function at $39 \%$ of the normal, which increases to $64 \%$ upon ivacaftor. Lastly, D537N demonstrated a defective maturation, which suggests that this mutant affects protein folding and is probably associated with a defective number of CFTR proteins at the apical membrane. The patient's NPD profile demonstrated residual $\mathrm{Cl}^{-}$activity but an increased response to Amiloride indicative of CFTR dysfunction, yielding a functional profile similar to F1052V.

These observations lead to general considerations in the context of unconcluded diagnosis after NBS. Although ICM may provide very valuable insight if abnormal given its specificity, it is not sensitive enough to detect variants which retain activity above $20 \%$ of the WT. In patients carrying variants with residual function between 10 and $20 \%$ of CFTR activity relative to wild-type, CFTR functional assays in the respiratory mucosa are more sensitive. This range of activity is often associated with variable expressivity and penetrance [40]. Indeed, as CFTR activity increases, the relation with ion transport in the respiratory and the sweat epithelium is nonlinear and may be also influenced by environmental factors or other ion transporter defects. In this context, evidence of an elevated $\triangle$ Amiloride response suggests CFTR dysfunction with residual or even normal $\mathrm{Cl}^{-}$transport. The threshold to discriminate between CF and CFTR-RD is currently unknown, but it is likely that a residual CFTR activity above $10 \%$ of the normal is consistent with the diagnosis of CFTR-RD [41].

Hence, our study helps refine the strategy of diagnosis in these unconcluded cases at NBS. If the genotype combines a CF-causing variant in trans of a VVCC, this indicates a high likelihood to evolve towards CF or a CFTR-RD, even if the probability cannot be stated because the penetrance of CF or CFTR-RD has not been defined for most of the variants. Functional measurement should be performed to characterize a VUS, and may be also proposed to assess the functional impact of a variant associated with a CFTR-RD, whose penetrance for $\mathrm{CF}$ is unknown. If ICM is abnormal this indicates 
a CFTR activity below 20\%. If it is normal, it does not rule out a genotype with residual activity, that could be compatible with an evolution toward a CFTR-RD. In this respect, CFTR activity should be investigated in the respiratory mucosa of the patient. If CFTR activity is decreased, this does not mean that those patients will have a $100 \%$ probability to develop symptoms later in life, in adulthood or even at older ages, but a decreased CFTR activity is clearly a sign of vigilance requiring continued follow-up in a CF center and preventive care. As NPD can be challenging in babies, a potential interesting test might be the evaluation of CFTR activity in primary nasal cells collected by a simple nasal brushing, which may be more feasible in young patients. CFTR activity in this model was shown to be correlated to in vivo NPD and might therefore provide reliable insight $[42,43]$. These primary cultures could also be used to test the correction of CFTR function by modulators and help to tailor the potential treatment of patients considered at risk to develop $\mathrm{CF}$, early in life.

\section{Conclusion}

It is crucial to detect which children with an unconclusive diagnosis at NBS are at increased likelihood for conversion to CF or CFTR-RD in order to best guide care and follow-up. We show here that combination of clinical follow-up, comprehensive CFTR gene analysis, in vitro and in vivo functional evaluation help reclassify all these children with inconclusive diagnosis at NBS.

Supplementary Fig. S1. Intestinal Short-circuit Current of a rectal biopsy from a subject carrying the F508del/TG11T5 genotype. Change in the Short-circuit Current (Isc) after sequential addition of different pharmacological agonists: Amiloride $100 \mu \mathrm{M}$ (apical side); Forskolin $10 \mu \mathrm{M}$ and IBMx $100 \mu \mathrm{M}$ (apical and basal side); Genistein $10 \mu \mathrm{M}$ (apical and the basal side) Carbachol $100 \mu \mathrm{M}$ (basal side); Histamin $500 \mu \mathrm{M}$ (basal side).

\section{Declaration of Competing Interest}

\section{All authors have disclosed their conflict of Interest.}

\section{CRediT authorship contribution statement}

Aurelie Hatton: Writing - review \& editing. Anne Bergougnoux: Writing - review \& editing. Katarzyna Zybert: Writing review \& editing. Benoit Chevalier: Writing - review \& editing. Myriam Mesbahi: Writing - review \& editing. Jean Pierre Altéri: Writing - review \& editing. Katarzyna Walicka-Serzysko: Writing - review \& editing. Magdalena Postek: Writing - review \& editing. Magali Taulan-Cadars: Writing - review \& editing. Aleksander Edelman: Writing - review \& editing. Alexandre Hinzpeter: Writing - review \& editing. Mireille Claustres: Writing - review \& editing. Emmanuelle Girodon: Writing - review \& editing. Caroline Raynal: Writing - review \& editing. Isabelle Sermet-Gaudelus: Writing - review \& editing. Dorota Sands: Writing - review \& editing.

\section{Acknowledgments}

We thank all the patients and their parents for their participation in the study. We thank Souphatta Sasorith and Corinne Bareil for providing detailed data from CFTR-France.

Financial support by Agence de la BioMédecine Appel d'Offre Recherche "AMP, diagnostic Prénatal et diagnostic génétique" 2015.

\section{References}

[1] Munck A. Inconclusive diagnosis after newborn screening for cystic fibrosis. Int J Neonatal Screen 2020;6:19. doi:10.3390/ijns6010019.

[2] Munck A Southern KW, Castellani C, de Winter-de Groot KM, Gartner S, Kashirskaya N, et al. Defining key outcomes to evaluate performance of newborn screening programmes for cystic fibrosis. J Cyst Fibros 2021. doi:10.1016/ j.jcf.2021.02.006.

[3] Audrézet MP, Munck A. Newborn screening for CF in France: An exemplary national experience. Arch Pediatr Feb 2020;27(Suppl 1) eS35-eS40. doi:10.1016 S0929-693X(20)30049-X.

[4] Kharrazi M, Yang J, Bishop T, Lessing S, Young S, Graham S, Pearl M, Chow H, Ho T, Currier R, Gaffney L, Feuchtbaum L. California cystic fibrosis newborn screening consortium. Newborn screening for cystic fibrosis in California. Pediatrics 2015;136(6):1062-72. doi:10.1542/peds.2015-0811.

[5] Salinas DB, Sosnay PR, Azen C, Young S, Raraigh KS, Keens TG, Kharrazi M. Benign outcome among positive cystic fibrosis newborn screen children with non-CF-causing variants. J Cyst Fibros 2015;14(6):714-19. doi:10.1016/j.jcf.2015. 03.006 .

[6] Salinas DB, Sosnay PR, Azen C, Young S, Raraigh KS, Keens TG, Kharrazi M. Benign and deleterious cystic fibrosis transmembrane conductance regulator mutations identified by sequencing in positive cystic fibrosis newborn screen children from California. PLoS One 2016;11(5):e0155624 23. doi:10.1371/journal. pone.0155624.

[7] Groves T, Robinson P, Wiley V, Fitzgerald DA. Long-term outcomes of children with intermediate sweat chloride values in infancy. J Pediatr 2015;166:146974 e1-3. doi:10.1016/j.jpeds.2015.01.052.

[8] Ren CL, Fink AK, Petren K, Borowitz DS, McColley SA, Sanders DB, et al. Outcomes of infants with indeterminate diagnosis detected by cystic fibrosis newborn screening. Pediatrics 2015;135:e1386-92. doi:10.1542/peds.2014-3698.

[9] Terlizzi V, Mergni G, Buzzetti R, Centrone C, Zavataro L, Braggion C. Cystic fibrosis screen positive inconclusive diagnosis (CFSPID): experience in Tuscany, Italy. J Cyst Fibros 2019;18:484-90. doi:10.1016/j.jcf.2019.04.002.

[10] Ooi CY, Castellani C, Keenan K, Avolio J, Volpi S, Boland M, et al. Inconclusive diagnosis of cystic fibrosis after newborn screening. Pediatrics 2015;135:e1377-85. doi:10.1542/peds.2014-2081.

[11] Salinas DB, Azen C, Young S, Keens TG, Kharrazi M, Parad RB. Phenotypes of California CF newborn screen-positive children with CFTR 5T Allele by TG repeat length. Genet Test Mol Biomarkers 2016;20:496-503. doi:10.1089/gtmb. 2016.0102

[12] Munck A, Bourmaud A, Bellon G, Picq P, Farrell PM. Phenotype of children with inconclusive cystic fibrosis diagnosis after newborn screening. Pediatr Pulmonol 2020;55:918-28. doi:10.1002/ppul.24634

[13] Ooi CY, Sutherland R, Castellani C, Keenan K, Boland M, Reisman J, et al. Immunoreactive trypsinogen levels in newborn screened infants with an inconclusive diagnosis of cystic fibrosis. BMC Pediatr 2019;19:369. doi:10.1186/ s12887-019-1756-4

[14] Chudleigh J, Chinnery H. Psychological impact of NBS for CF. Int J Neonatal Screen 2020;6:27. doi:10.3390/ijns6020027.

[15] Sosnay PR, Siklosi KR, Van Goor F, Kaniecki K, Yu H, Sharma N, et al. Defining the disease liability of variants in the cystic fibrosis transmembrane conductance regulator gene. Nat Genet 2013;45:1160-7. doi:10.1038/ng.2745.

[16] Sermet-Gaudelus I, Brouard J, Audrézet M-P, Couderc Kohen L, Weiss L, Wizla N, et al. Guidelines for the clinical management and follow-up of infants with inconclusive cystic fibrosis diagnosis through newborn screening. Arch Pediatr 2017;24:e1-14. doi:10.1016/j.arcped.2017.07.015

[17] Boussaroque A, Audrézet M-P, Raynal C, Sermet-Gaudelus I, Bienvenu T, Férec $C$, et al. Penetrance is a critical parameter for assessing the disease liability of CFTR variants. J Cyst Fibros 2020;19:949-54. doi:10.1016/j.jcf.2020. 03.019.

[18] Thauvin-Robinet C, Munck A, Huet F, Génin E, Bellis G, Gautier E, et al. The very low penetrance of cystic fibrosis for the $\mathrm{R} 117 \mathrm{H}$ mutation: a reappraisal for genetic counselling and newborn screening. J Med Genet 2009;46:752-8. doi:10.1136/jmg.2009.067215

[19] Cuppens H, Lin W, Jaspers M, Costes B, Teng H, Vankeerberghen A, et al. Polyvariant mutant cystic fibrosis transmembrane conductance regulator genes. The polymorphic ( $\mathrm{Tg}$ )m locus explains the partial penetrance of the T5 poly morphism as a disease mutation. J Clin Invest 1998;101:487-96. doi:10.1172/ JCI639.

[20] Sobczyńska-Tomaszewska A, Ołtarzewski M, Czerska K, WertheimTysarowska K, Sands D, Walkowiak J, et al. Newborn screening for cystic fibrosis: polish 4 years' experience with CFTR sequencing strategy. Eur J Hum Genet 2013;21:391-6. doi:10.1038/ejhg.2012.180.

[21] Sands D, Zybert K, Mierzejewska E, Ołtarzewski M. Diagnosing cystic fibrosis in newborn screening in Poland - 15 years of experience. Dev Period Med 2015;19:16-24

[22] Castellani C, Conway S, Smyth AR, Stern M, Elborn JS. Standards of care for cystic fibrosis ten years later. J Cyst Fibros 2014;13(Suppl 1):S1-2. doi:10.1016/ j.jcf.2014.03.008

[23] Baux D, Vaché C, Blanchet C, Willems M, Baudoin C, Moclyn $M$, et al. Combined genetic approaches yield a $48 \%$ diagnostic rate in a large cohort of French hearing-impaired patients. Sci Rep 2017;7:16783. doi:10.1038/ s41598-017-16846-9.

[24] Tsui LC. Mutations and sequence variations detected in the cystic fibrosis transmembrane conductance regulator (CFTR) gene: a report from the cystic 
fibrosis genetic analysis consortium. Hum Mutat 1992;1:197-203. doi:10.1002/ humu.1380010304.

[25] Claustres M, Thèze $C$, des Georges M, Baux D, Girodon E, Bienvenu T, et al. CFTR-France, a national relational patient database for sharing genetic and phenotypic data associated with rare CFTR variants. Hum Mutat 2017;38:1297315. doi:10.1002/humu.23276.

[26] Sasorith S, Baux D, Bergougnoux A, Paulet D, Lahure A, Bareil C, et al. The CYSMA web server: An example of integrative tool for in silico analysis of missense variants identified in Mendelian disorders. Hum Mutat 2020;41:375-86. doi:10.1002/humu.23941.

[27] Baux D, Van Goethem C, Ardouin O, Guignard T, Bergougnoux A, Koenig M, et al. MobiDetails: online DNA variants interpretation. Eur J Hum Genet 2021;29:356-60. doi:10.1038/s41431-020-00755-z.

28] Fanen P, Clain J, Labarthe R, Hulin P, Girodon E, Pagesy P, et al. Structurefunction analysis of a double-mutant cystic fibrosis transmembrane conductance regulator protein occurring in disorders related to cystic fibrosis. FEBS Lett 1999;452:371-4. doi:10.1016/s0014-5793(99)00647-X.

[29] Pranke I, Bidou L, Martin N, Blanchet S, Hatton A, Karri S, et al. Factors influencing readthrough therapy for frequent cystic fibrosis premature termination codons. ERJ Open Res 2018;4. doi:10.1183/23120541.00080-2017.

[30] Derichs N, Sanz J, Von Kanel T, Stolpe C, Zapf A, Tümmler B, et al. Intestinal current measurement for diagnostic classification of patients with questionable cystic fibrosis: validation and reference data. Thorax 2010;65:594-9. doi:10.1136/thx.2009.125088.

[31] Sermet-Gaudelus I, Girodon E, Roussel D, Deneuville E, Bui S, Huet F, et al. Measurement of nasal potential difference in young children with an equivocal sweat test following newborn screening for cystic fibrosis. Thorax 2010;65:539-44. doi:10.1136/thx.2009.123422.

[32] Bonini J, Varilh J, Raynal C, Thèze C, Beyne E, Audrezet MP, et al. Small-scale high-throughput sequencing-based identification of new therapeutic tools in cystic fibrosis. Genet Med 2015;17:796-806. doi:10.1038/gim.2014.194.

[33] Bergougnoux A, Délétang K, Pommier A, Varilh J, Houriez F, Altieri JP, et al. Functional characterization and phenotypic spectrum of three recurrent disease-causing deep intronic variants of the CFTR gene. J Cyst Fibros 2019;18:468-75. doi:10.1016/j.jcf.2018.10.012.
[34] Dickinson P, Smith SN, Webb S, Kilanowski FM, Campbell IJ, Taylor MS, et al. The severe G480C cystic fibrosis mutation, when replicated in the mouse demonstrates mistrafficking, normal survival and organ-specific bioelectrics. Hum Mol Genet 2002;11:243-51. doi:10.1093/hmg/11.3.243.

[35] Terlizzi V, Carnovale V, Castaldo G, Castellani C, Cirilli N, Colombo C, et al. Clinical expression of patients with the D1152H CFTR mutation. J Cyst Fibros 2015;14:447-52. doi:10.1016/j.jcf.2014.12.012.

[36] Minso R, Schulz A, Dopfer C, Alfeis N, van Barneveld A, MakartianGyulumyan L, et al. Intestinal current measurement and nasal potential difference to make a diagnosis of cases with inconclusive CFTR genetics and sweat test. BMJ Open Respir Res 2020;7. doi:10.1136/bmjresp-2020-000736.

[37] Aalbers BL, Yaakov Y, Derichs N, Simmonds NJ, De Wachter E, Melotti P, et al. Nasal potential difference in suspected cystic fibrosis patients with $5 \mathrm{~T}$ polymorphism. J Cyst Fibros 2020;19:627-31. doi:10.1016/j.jcf.2019.07.001.

[38] Boussaroque A, Bergougnoux A, Raynal C, Audrézet M-P, Sasorith S, Férec C, et al. Pitfalls in the interpretation of CFTR variants in the context of incidental findings. Hum Mutat 2019;40:2239-46. doi:10.1002/humu.23884.

[39] Mesbahi M, Shteinberg M, Wilschanski M, Hatton A, Nguyen-Khoa T, Friedman $\mathrm{H}$, et al. Changes of CFTR functional measurements and clinical improvements in cystic fibrosis patients with non p.Gly551Asp gating mutations treated with ivacaftor. J Cyst Fibros 2017;16:45-8. doi:10.1016/j.jcf.2016.08.006.

[40] McCague AF, Raraigh KS, Pellicore MJ, Davis-Marcisak EF, Evans TA, Han ST, et al. Correlating cystic fibrosis transmembrane conductance regulator function with clinical features to inform precision treatment of cystic fibrosis. Am J Respir Crit Care Med 2019;199:1116-26. doi:10.1164/rccm.201901-01450C.

[41] Davis Pamela B. Cystic fibrosis. Pediatr Rev 2001;22(8):257-64 Aug. doi:10. 1542/pir.22-8-257.

[42] Pranke IM, Hatton A, Simonin J, Jais JP, Le Pimpec Barthes F, Carsin A, et al. Correction of CFTR function in nasal epithelial cells from cystic fibrosis patients predicts improvement of respiratory function by CFTR modulators. Sci Rep 2017;7:7375. doi:10.1038/s41598-017-07504-1.

[43] Noel S, Servel N, Hatton A, Golec A, Rodrat M, et al. Correlating genotype with phenotype using CFTR-mediated whole-cell $\mathrm{Cl}^{-}$currents in human nasal epithelial cells. J Physiol 2021 Epub Ahead of Print. 